\title{
ANÁLISE DA CONTRIBUIÇÃO DA INTEGRAÇÃO DE SISTEMAS DE INFORMAÇÃO NAS ORGANIZAÇÕES: UM ESTUDO EXPLORATÓRIO COM GESTORES DE TIC EM PERNAMBUCO
}

\section{ANALYSIS OF THE CONTRIBUTION OF INFORMATION SYSTEMS INTEGRATION IN ORGANIZATIONS: AN EXPLORATORY STUDY WITH ICT MANAGERS IN PERNAMBUCO}

\author{
Anderson Lucas Carneiro de Lima da Silva*E-mail: andersonlucas12@hotmail.com \\ Suzana de França Dantas Daher* E-mail: suzanadaher@cdsid.org.br \\ Lúcio Camara e Silva* E-mail: luciocsilva@gmail.com \\ *Universidade Federal de Pernambuco (UFPE), Recife, PE
}

\begin{abstract}
Resumo: Uma vez que os investimentos em Tecnologia da Informação e Comunicação (TIC) têm crescido dentro das organizações, e a estes estão associados desafios quanto a identificação e quantificação dos retornos, estudos neste sentido se tornam de grande importância. Uma tendência de investimentos nos últimos anos é a adoção de Sistemas de Informação (SIs) integrados ou a integração dos já existentes nas empresas. As razões que levam os gestores a adotarem tais formas de investimentos em TIC são, de maneira geral, a busca por melhorias de desempenho e vantagem competitiva, tendo em vista, os ambientes de negócio cada vez mais dinâmicos. Diante disto, o presente trabalho teve como objetivo investigar o impacto da integração de Sls na competitividade das empresas pernambucanas de diversos setores. Para tanto, uma coleta de dados foi realizada por meio um questionário elaborado nos formatos físico e online e enviado às empresas, além de testes de hipótese com os dados obtidos. Os resultados identificados demonstraram a influência da integração sobre a compreensão do negócio, aprendizado dos funcionários e inovação, enquanto que, nenhuma relação pôde ser constatada no que se refere a custos e controle de atividades.
\end{abstract}

Palavras-Chave: Investimentos. TIC. Integração de SIs. Estratégia. Competitividade.

\begin{abstract}
Since investments in Information and Communication Technology (ICT) have grown within organizations, and these are associated with challenges in identifying and quantifying returns, studies over this theme become more importance. Investment trends in recent years are the adoption of integrated Information Systems (ISs) or the integration of existing ones in companies. The reasons that lead managers to adopt those ICT investments are, in general, the pursuit of performance improvements and competitive advantage, considering the existence of dynamic business environments even more competitive. Thus, the objective of this study is investigate the impact of IS integration on the competitiveness of Pernambuco companies in different sectors. Therefore, a data collection was performed through a questionnaire elaborated in physical and online formats and sent to companies. After that, hypothesis tests were performed with the data obtained. The results identified demonstrated the influence of integration on business understanding, employee learning and innovation, whereas no relationship could be verified with regard to costs and activity control.
\end{abstract}

Keywords: Investments. ICT. IS integration. Strategy. Competitiveness.

\section{INTRODUÇÃO}

Investimentos em Tecnologia da Informação e Comunicação (TIC) dentro do 
contexto organizacional têm consumindo parte considerável dos recursos disponíveis (KHALLAF, 2012). A medida que estes investimentos representam oportunidades de sobrevivência e de vantagem competitiva, os mesmos também oferecem muitos desafios. Da literatura, tem-se observado evidências tanto positivas quanto negativas dos investimentos em TIC, tornando inconclusivo a influência destes sobre a performance organizacional (KHALLAF, 2012). Assim, buscar soluções eficazes para que a TIC apoie a organização se configura como um processo complexo que vai desde a escolha da forma do investimento até o método de avaliação dos benefícios e dos retornos tangíveis e intangíveis obtidos. Quanto aos métodos para avaliação, a aplicação exclusiva de medidas financeiras pode não ser adequada, uma vez que pode criar percepções enganosas sobre a eficácia dos investimentos (BALASUBRAMANIAN; KULATILAKA; STORCK, 2000; JOSHI; PANT, 2008; KHALLAF, 2012; PERERA; 2012; KAPLAN; NORTON, 2001).

Avanços recentes na área de TIC têm proporcionando o desenvolvimento e disponibilização de ampla variedade de Sistemas de Informação (Sls) com as mais variadas funções capazes de atender múltiplas demandas organizacionais. Dentro deste contexto, uma ação que muitas organizações têm adotado quanto ao uso de seus Sls é a aplicação da integração dos mesmos ou aquisição de sistemas já integrados. Dentre outros benefícios, têm-se o compartilhamento mais amplo de informações e integração eficiente entre áreas funcionais de uma empresa e entre organizações membro da cadeia de suprimentos. Entretanto, como os demais tipos de investimentos em TIC, a integração de Sls também apresenta complexidades, sendo um processo que envolve vários critérios e condições específicas a serem considerados para o seu efetivo êxito. (ARSHAH; DESA; HUSSIN, 2008; LI; SU, 2001; HAMMER, 2001; MAIGA; NILSSON; AX, 2015; MÖLLER; FRANK; CORTIMIGLIA, 2014).

Diante disto, o presente estudo buscou identificar a relação entre o nível de integração de Sls das organizações e os retornos obtidos decorrentes dos investimentos realizados na área de TIC através de uma analise exploratória com dados de empresas do estado de Pernambuco. Trata-se de uma continuação dos trabalhos desenvolvidos por Silva, Daher e Silva (2015) no qual foi identificado o perfil dos investimentos realizados no estado de Pernambuco quanto à TIC, e por Silva, Daher e Silva (2016) no qual a influência da integração de Sls sobre alguns 
ganhos organizacionais foi analisada. Aqui, para tanto, cinco aspectos em específicos foram analisados, são eles: compreensão do negócio, aprendizado dos funcionários, inovação, controle de atividades e redução de custos. Testes de hipóteses com as amostras coletadas foram realizados, e, a partir dos resultados obtidos e da revisão da literatura realizada, procurou-se compreender algumas relações identificadas entre o nível de integração dos Sls das organizações e (1) os ganhos com compreensão do negócio, (2) aprendizado dos funcionários e (3) inovação.

Este artigo é organizado com inicial apresentação do referencial teórico e uma breve revisão da literatura. Segue-se, então, com a explicação da metodologia do estudo e apresentação dos resultados obtidos. Por fim, tem-se as discussões sobre os resultados e a conclusão final deste trabalho.

\title{
2 INVESTIMENTOS EM TECNOLOGIA DA INFORMAÇÃO E COMUNICAÇÃO (TIC)
}

A Tecnologia da Informação e Comunicação (TIC), conforme apontado por Beal (2008, p.17), pode ser definida como

\begin{abstract}
soluções sistematizadas baseadas no uso de métodos, recursos de informática, de comunicação, de multimídia que visam resolver problemas relativos à geração, armazenamento, veiculação, processamento e reprodução de dados e a subsidiar processos que convertem dados em informação.
\end{abstract}

Acerca de tais recursos, os investimentos realizados nos últimos anos têm se tornado cada vez mais presentes dentro do ambiente organizacional, consumindo, portanto, parte considerável do capital das empresas. Contudo, a decisão de investir em TIC, bem como, a forma como este se dará, tendo em vista o seu êxito, nem sempre é considerada uma tarefa simples, isto porque, enquanto que os custos associados aos investimentos serem facilmente quantificáveis, os retornos sobre estes, nem sempre o são, tornando o processo de investimento carente de cuidados e atenção (BALASUBRAMANIAN; KULATILAKA; STORCK, 2000; CHOU; CHOU; TZENG, 2006).

Os investimentos em TIC podem ser realizados em duas perspectivas, 
conforme apontado por Khallaf (2012), seja com foco interno, ou com foco externo à organização. Estes investimentos são motivados pela busca do aumento da eficiência dos processos de negócios e vantagem competitiva. Deste modo, no âmbito interno, o que os gestores almejam é a redução de custos, aumento de qualidade e da velocidade de operações, além do ganho em flexibilidade. Por sua vez, no âmbito externo, o foco é na melhoria do relacionamento com o cliente, aumento da satisfação dos mesmos, análise sobre os concorrentes, novas oportunidades e possíveis ameaças vindas do ambiente externo.

Outro ponto associado aos investimentos em TIC é a escolha dos indicadores de desempenho para a avaliação dos ganhos oriundos da TIC. Apesar dos indicadores financeiros serem comumente utilizados para avaliação de ganhos, estes nem sempre são apropriados para os investimentos em TIC, uma vez que os ganhos com tais investimentos podem não ser sempre perceptíveis aos gestores, isto porque os benefícios dos investimentos em TIC vão além de aspectos econômicos, alcançando, também, aspectos intangíveis. Desta forma, uma abordagem exclusivamente financeira pode interferir negativamente na execução dos investimentos como também na compreensão dos mesmos e de seus benefícios, e, portanto, impactando no papel da TIC dentro das organizações (KHALLAF, 2012; KAPLAN; NORTON, 2001; BHARADWAJ; BHARADWAJ; KONSYNSKI, 1999; SILVA; DAHER; SILVA, 2015).

Ainda com relação aos investimentos em TIC, tem-se o chamado paradoxo da produtividade. Este foi primeiramente analisado por Solow (1987) como resultado da percepção de que o crescimento do uso de recursos de TIC, em certa medida, era acompanhado pela queda da produtividade do trabalho, pondo em dúvida os benefícios do uso de tais recursos e estimulando o desenvolvimento de pesquisas na área. De acordo com Brynjolfsson (1993), alguns fatores podem contribuir para o paradoxo da produtividade, como, por exemplo: a medição incorreta, incapaz de avaliar os benefícios decorrentes da TIC; a distribuição dos benefícios destes recursos com a dissipação dos lucros, entre outros. Contudo, mesmo havendo muitos estudos com evidências do paradoxo, novas pesquisas desenvolvidas nos últimos anos têm indicado o contrario, tendendo a concluir a não existência deste. De maneira geral, a gestão dos investimentos deve ser realizada de maneira cautelosa a fim de minimizar os riscos com estes (BROWN, 2015; HAJLI; SIMS, 
2015).

Desta forma, uma abordagem mais eficiente no que tange à análise de investimentos em TIC deve considerar não só os aspectos tangíveis, como, também, os técnicos, intangíveis e a visão de todos os stakeholders envolvidos (AZADEH; KERAMATI; SONGHORI, 2009; MIRANI; LEDERER, 1998; CHOU; CHOU; TZENG, 2006). Neste sentido, além dos aspectos financeiros, a seleção dos investimentos deve considerar tanto as características organizacionais, quanto o ambiente competitivo, os envolvidos na organização e critérios específicos para cada projeto. Por sua vez, para acompanhamento e controle dos projetos, é importante que indicadores variados sejam definidos para identificação e correção de desvios de objetivos e maior comprometimento da área de TIC. Através desse novo modelo de tomada de decisão, torna-se possível reduzir os riscos com os investimentos realizados nesta área, além de aumentar a percepção dos gerentes com os ganhos gerados por esta área (AZADEH; KERAMATI; SONGHORI, 2009; CHOU; CHOU; TZENG, 2006; KHALLAF, 2012).

\section{INTEGRAÇÃO DE SISTEMAS DE INFORMAÇÃO}

Os recentes avanços tecnológicos têm proporcionado o desenvolvimento de variados tipos de sistemas de informação (SIs) com múltiplas funções utilizados pelas empresas a fim de atenderem necessidades operacionais. Por sistemas de informação, entende-se o conjunto organizado de hardware, software, rede, dados e pessoas, sendo este último elemento o mais importante em Sls. Mudanças, entretanto, das organizações por meio do crescimento das mesmas, com ampliação do número de seus clientes, fornecedores, produtos, entre outros, além do dinamismo do cenário econômico cada vez mais competitivo, têm exigido alterações quanto ao uso dos Sls dentro das organizações. Assim, o que antes funcionava como sistemas isolados se torna inadequado, e, em alguns casos, inviável à nova realidade das empresas, tendo em vista a sobrevivência e competitividade das mesmas, demandando, assim, a integração de tais recursos. Esta última, por sua vez, consiste basicamente na interconexão e na intercomunicação de sistemas com a combinação dos seus respectivos bancos de dados (LI; SU, 2001; LITAN et al., 2011). 
É comum organizações disporem de Sls para cada área funcional ou alguns processos específicos, sendo, em alguns casos, cada Sls utilizado de maneira isolada para a obtenção, transformação, geração e armazenamento de dados que sejam críticos para suas respectivas operações. Entretanto, tal separação entre os Sls dentro da própria organização cria ilhas isoladas de dados, deixando de fornecer dados que poderiam ser úteis para outras áreas funcionais, caso uma integração interna houvesse sido realizada. Ainda nesse sentido, a integração pode ser feita de maneira externa, e, nesse caso, considerando sistemas de empresas diferentes. Deste modo, de maneira colaborativa, ambas empresas podem se beneficiar por meio de ganho em flexibilidade, capacidade de respostas, entre outros. Em suma, a integração serve como forma de amparar as necessidades organizacionais, acelerando o tempo de respostas às solicitações dos usuários e com o fornecimento de informações e dados úteis sem muitos limites e restrições. Tal constatação reforça, portanto, a ideia de que dispor de Sls para gerenciamento de informações não garante por si só eficácia. (VARGA; ĆURKO, 2012; RAJAGURU; MATANDA, 2013; TOMICIC-PUPEK; DOBROVIC; FURJAN, 2012; ARSHAH; DESA; HUSSIN, 2008; MÖLLER et al. 2013).

À medida que as organizações crescem e se tornam mais complexas, o processo de integração de Sls se torna mais necessário, fazendo com que características da integração precisem ser atendidas com maior rigor. Algumas dessas características são: modularidade, coerência, interoperabilidade vertical, interoperabilidade horizontal, integridade, transparência, disponibilidade, funcionalidade, confiabilidade, usabilidade, eficiência, sustentabilidade e portabilidade (TOMICIC-PUPEK; DOBROVIC; FURJAN, 2012). Tais características têm influência na seleção do projeto de integração de SIs de modo que deve ser acompanhado da análise dos ambientes internos e externos da organização, a fim do alinhamento com seus os objetivos estratégicos. Ou seja, a integração e atendimento de tais características devem ser feitos de forma planejada para o efetivo alcance do seu propósito, devendo ser dado considerado destaque à fase de implementação, uma das mais críticas para o sucesso de Sls integrados (MÖLLER; FRANK; CORTIMIGLIA, 2014).

Mediante isso, estudos a fim de avaliar a eficácia da integração de Sls têm sido desenvolvidos em diversas nações e em diversos setores da economia. Roberts 
e Grover (2012), por exemplo, analisaram o efeito da integração tanto interna quanto externa em empresas dos Estados Unidos, constatando o impacto desta sobre a melhoria da coordenação interfuncional das organizações e da capacidade de resposta aos clientes. Já o trabalho de Darmawan Raymond (2015) teve como foco a cadeia de suprimentos do setor automotivo na Indonésia e constatou redução no tempo de entrega de itens, impactando diretamente no numero de atrasos, uma vez que a integração proporcionou o fornecimento eficiente de informações acerca do estoque aos parceiros comerciais. Shanahan et al. (2014), por sua vez, analisou o impacto da integração no setor de serviços americano, mais especificamente, o de clínicas médicas. Neste caso, os resultados obtidos foram o de ganhos em eficiência e qualidade, e, portanto, aumento da satisfação dos clientes.

Todavia, evidências negativas acerca da integração também foram observadas em estudos. Charoen (2015) em seu trabalho destacou o surgimento de conflitos internos e desconfiança decorrentes da estrutura do sistema integrado que permite que erros sejam facilmente identificados, bem como os seus responsáveis. Por sua vez, Sun e Ifeanyi (2015) constataram que a integração incorre em crescimento de custos de manutenção do sistema, além de riscos associados aos dados. Por fim, Waring e Skoumpopoulou (2012) evidenciou que a adoção de um sistema integrado numa universidade no Reino Unido criou uma estrutura enrijecida, dificultando o processo de inovação da instituição.

Desta forma, até onde se têm conhecimento, este trabalho se difere dos demais, considerando o impacto da integração de Sls sobre investimentos realizados tendo em vista a compreensão do negócio, aprendizado dos funcionários, inovação, redução de custos e controle das atividades nos três setores da economia (industrial, de comércio e serviços) do estado de Pernambuco. Neste estudo, testes de hipóteses com dados coletados de diversas empresas são realizados a fim de trazer mais evidências sobre o papel que a integração de Sls exerce nos retornos sobre investimentos estratégicos realizados pelas organizações.

\section{METODOLOGIA DA PESQUISA}

Foram coletados dados através de um questionário elaborado nas versões física e online enviados para empresas do estado de Pernambuco dos setores 
industrial, de serviços e comércio. O questionário consistiu de 78 questões, sendo seis abertas e 72 de múltipla escolha. Para a versão online, foi utilizado o Google Forms. Todas as respostas obtidas foram salvas e analisadas no software Microsoft Excel. Para este estudo, foram considerados os dados obtidos de 33 gestores de TIC.

O estudo foi divido em cinco etapas nas quais foram realizados testes de hipótese acerca da relação do nível de integração dos sistemas de informação presentes nas organizações com os ganhos percebidos com investimentos em TIC no que diz respeito a: compreensão do negócio, aprendizado dos funcionários, inovação, controle de atividades e redução de custos.

A prova de Fisher foi a análise estatística escolhida para a realização dos testes. Foi aplicada ainda a modificação de Tocher, em função da menor frequência observada ter sido maior que zero. $\mathrm{H}_{0}$ e $\mathrm{H}_{1}$ representaram a hipótese nula e a hipótese alternativa, respectivamente, enquanto que, para a representação das probabilidades do teste de Fisher calculadas, foram utilizados: $p_{1}=$ probabilidade para tabela sem a modificação de Tocher; e $\mathrm{p}_{2}=$ probabilidade para tabela com a modificação de Tocher; e $p_{t}=$ soma de $p_{1}$ e $p_{2}$. O nível de significância adotado foi de 0,05 , sendo representado por $\alpha$. Os testes realizados foram unilaterais. A Figura 1 apresenta as relações avaliadas neste estudo. 
Figura 1 - Relação de integração de Sistemas de Informação com retornos sobre investimentos em Tecnologia da Informação e Comunicação com compreensão de negócio, aprendizado dos funcionários, inovação, controle de atividades e redução de custos

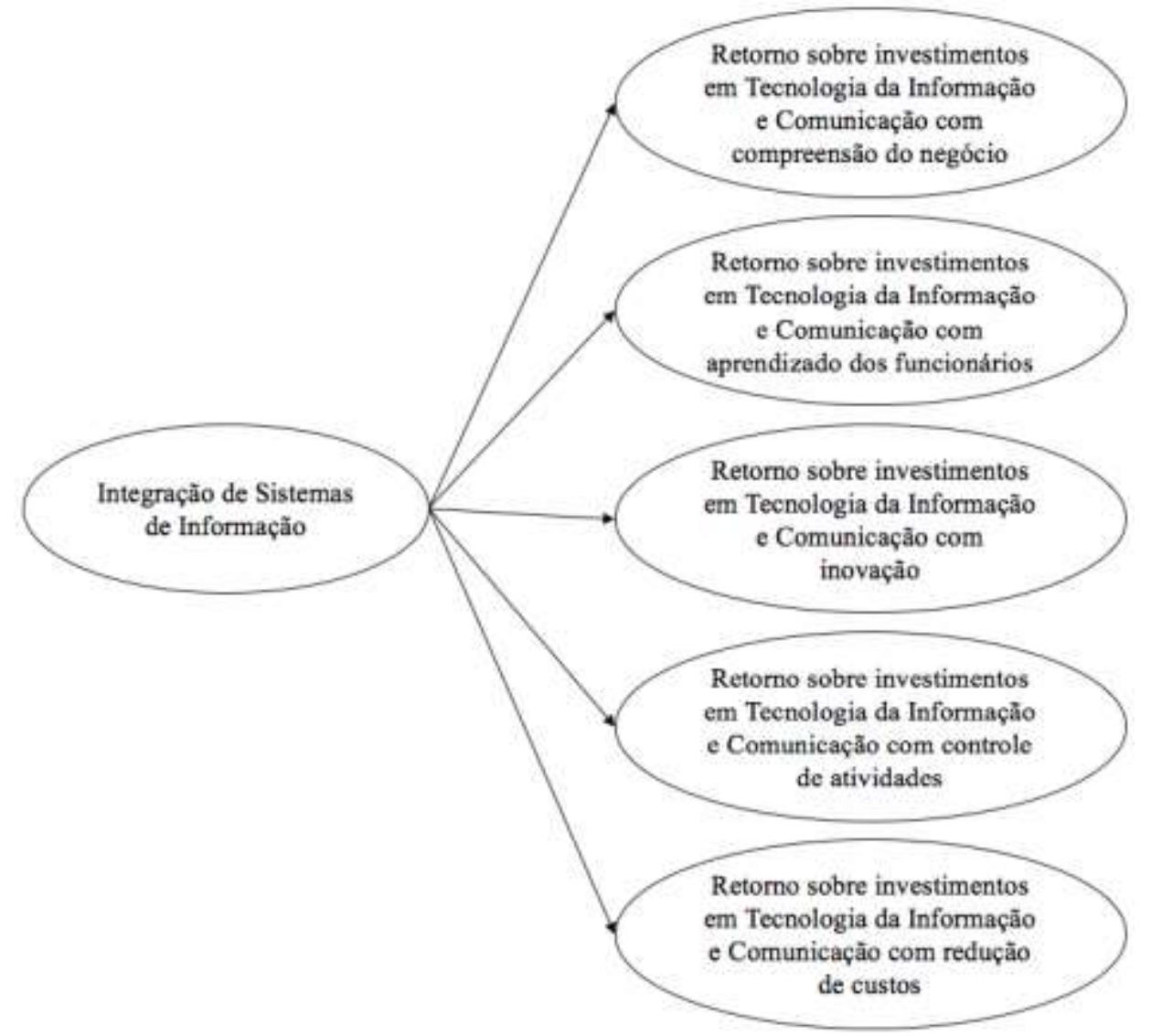

\section{ANÁLISE DOS DADOS}

Quatro variáveis foram utilizadas para as análises realizadas, foram elas: nível de integração de sistemas de informação, ganho com compreensão do negócio, ganho com aprendizados dos funcionários e ganho com inovação.

\subsection{Relação entre integração dos sistemas existentes com compartilhamento de dados e aplicações versus Ganho com compreensão do negócio com base nos investimentos feitos no ano anterior em TIC}

Nesta etapa da pesquisa foram analisadas as variáveis "nível de integração dos sistemas existentes com compartilhamento de dados e aplicações" e "nível de ganho com compreensão do negócio com base nos investimentos feitos no ano anterior em TIC". As hipóteses consideradas foram as seguintes: 
- $\mathrm{H}_{0}$ : o nível de integração de sistemas de informação não interfere no ganho com compreensão do negócio;

- $H_{1}$ : a integração de sistemas de informação influencia o ganho com compreensão do negócio.

Os valores das frequências observadas para a relação considerada aqui são apresentados a seguir na Tabela 1.

Tabela 1 - Frequências observadas para o tipo de ganho com compreensão do negócio associado ao nível de integração dos sistemas de informação das empresas pernambucanas

\begin{tabular}{|c|c|c|c|}
\hline & $\begin{array}{c}\text { Pequeno ou médio } \\
\text { ganho com } \\
\text { compreensão do } \\
\text { negócio }\end{array}$ & $\begin{array}{c}\text { Grande ou enorme } \\
\text { ganho com } \\
\text { compreensão do } \\
\text { negócio }\end{array}$ & Total \\
\hline $\begin{array}{c}\text { Nenhuma, pouca ou } \\
\text { razoável integração } \\
\text { dos sistemas }\end{array}$ & 10 & 1 & 11 \\
\hline $\begin{array}{c}\text { Boa ou total } \\
\text { integração dos } \\
\text { sistemas }\end{array}$ & 12 & 10 & 22 \\
\hline Total & 22 & 11 & 33 \\
\hline
\end{tabular}

Pela prova de Fisher, o valor da probabilidade de distribuição de frequências calculado foi $p_{1}=0,03675$. Entretanto, como a menor frequência observada foi maior que zero, a modificação de Tocher foi aplicada. Assim, a nova tabela de contingencia é apresentada na Tabela 2:

Tabela 2 - Frequências observadas para o tipo de ganho com compreensão do negócio associado ao nível de integração dos sistemas de informação das empresas pernambucanas com a modificação de Tocher

\begin{tabular}{|c|c|c|c|}
\hline & $\begin{array}{c}\text { Pequeno ou médio } \\
\text { ganho com } \\
\text { compreensão do } \\
\text { negócio }\end{array}$ & $\begin{array}{c}\text { Grande ou enorme } \\
\text { ganho com } \\
\text { compreensão do } \\
\text { negócio }\end{array}$ & Total \\
\hline $\begin{array}{c}\text { Nenhuma, pouca ou } \\
\text { razoável integração } \\
\text { dos sistemas }\end{array}$ & 11 & 0 & 11 \\
\hline $\begin{array}{c}\text { Boa ou total } \\
\text { integração dos } \\
\text { sistemas }\end{array}$ & 11 & 11 & 22 \\
\hline Total & 22 & 11 & 33 \\
\hline
\end{tabular}

Aqui, a probabilidade pela prova de Fisher decorrente da nova tabela foi $\mathrm{p}_{2}=$ 0,00364 . Como nesta tabela a menor frequência assumiu o valor de zero, não foi necessário aplicar a modificação de Tocher mais uma vez. Portanto, o valor final de probabilidade consistiu no somatório dos valores de probabilidade parciais, $\mathrm{p}_{1}$ e $\mathrm{p}_{2}$, 
sendo $p_{t}=0,4039$. Como $p_{t}<\alpha$, a hipótese nula foi rejeitada, indicando que o nível de integração entre os sistemas de informação interfere nos ganhos com compreensão do negócio.

\subsection{Relação entre Integração dos sistemas existentes com compartilhamento de dados e aplicações versus ganho com aprendizado dos funcionários com base nos investimentos feitos no ano anterior em TIC}

Na segunda etapa da pesquisa, a análise realizada considerou as seguintes variáveis: "nível de integração dos sistemas existentes com compartilhamento de dados e aplicações" e "nível de ganho com aprendizado dos funcionários com base nos investimentos feitos no ano anterior em TIC". Deste modo, as hipóteses consideradas foram:

- $\mathrm{H}_{0}$ : o nível de integração de sistemas de informação não interfere no ganho com aprendizado dos funcionários;

- $\mathrm{H}_{1}$ : a integração de sistemas de informação influencia o ganho com aprendizado dos funcionários.

A Tabela 3 apresenta as frequências observadas para as amostras consideradas nesta etapa do estudo.

Tabela 3 - Frequências observadas para o tipo de ganho com aprendizado dos funcionários associado ao nível de integração dos sistemas de informação das empresas pernambucanas

\begin{tabular}{|c|c|c|c|}
\hline & $\begin{array}{c}\text { Pequena ou médio } \\
\text { ganho com } \\
\text { aprendizado }\end{array}$ & $\begin{array}{c}\text { Grande ou enorme } \\
\text { ganho com } \\
\text { aprendizado }\end{array}$ & Total \\
\hline $\begin{array}{c}\text { Nenhuma, pouca ou } \\
\text { razoável integração } \\
\text { dos sistemas }\end{array}$ & 10 & 1 & 11 \\
\hline $\begin{array}{c}\text { Boa ou total } \\
\text { integração dos } \\
\text { sistemas }\end{array}$ & 11 & 11 & 22 \\
\hline Total & 21 & 12 & 33 \\
\hline
\end{tabular}

Pela aplicação da prova de Fisher nesta etapa, o valor de probabilidade calculado $p_{1}$ foi de 0,04009 . Contudo, de maneira análoga à etapa anterior, foi aplicada, também, a modificação de Tocher, sendo que a Tabela 4 apresenta as novas frequências. 
Tabela 4 - Frequências observadas para o tipo de ganho com aprendizado dos funcionários associado ao nível de integração dos sistemas de informação das empresas pernambucanas com a modificação de Tocher

\begin{tabular}{|c|c|c|c|}
\hline & $\begin{array}{c}\text { Pequena ou médio } \\
\text { ganho com } \\
\text { aprendizado }\end{array}$ & $\begin{array}{c}\text { Grande ou enorme } \\
\text { ganho com } \\
\text { aprendizado }\end{array}$ & Total \\
\hline $\begin{array}{c}\text { Nenhuma, pouca ou } \\
\text { razoável integração } \\
\text { dos sistemas }\end{array}$ & 11 & 0 & 11 \\
$\begin{array}{c}\text { Boa ou total } \\
\text { integração dos } \\
\text { sistemas }\end{array}$ & 10 & 12 & 22 \\
\hline Total & 21 & 12 & 33 \\
\hline
\end{tabular}

O novo valor de probabilidade $\mathrm{p}_{2}$ obtido foi de 0,00334 . Somando-se este com $\mathrm{p}_{1}$, o valor de $\mathrm{p}_{\mathrm{t}}$, aqui, foi igual a 0,04343, e, portanto, menor que o valor do nível de significância adotado de 5\%. Deste modo, pôde-se rejeitar a hipótese nula de que o nível de integração dos sistemas de informação não interfere no nível do ganho percebido com o aprendizado dos funcionários.

\subsection{Relação entre integração dos sistemas existentes com compartilhamento de dados e aplicações versus ganho com inovação com base nos investimentos feitos no ano anterior em TIC}

$\mathrm{Na}$ terceira etapa, foi analisado a relação entre as variáveis "nível de integração dos sistemas existentes com compartilhamento de dados e aplicações" e "nível de ganho com inovação com base nos investimentos feitos no ano anterior em TIC", tendo as hipóteses a seguir:

- $\mathrm{H}_{0}$ : o nível de integração de sistemas de informação não interfere no ganho com inovação;

- $\mathrm{H}_{1}$ : a integração de sistemas de informação influencia o ganho com inovação.

As frequências observadas para as amostras consideradas nesta etapa do estudo são apresentadas na Tabela 5. 
Tabela 5 - Frequências observadas para o tipo de ganho com inovação associado ao nível de integração dos sistemas de informação das empresas pernambucanas

\begin{tabular}{|c|c|c|c|}
\hline & $\begin{array}{c}\text { Pequeno ou médio } \\
\text { ganho com inovação }\end{array}$ & $\begin{array}{c}\text { Grande ou enorme } \\
\text { ganho com inovação }\end{array}$ & Total \\
\hline $\begin{array}{c}\text { Nenhuma, pouca ou } \\
\text { razoável integração } \\
\text { dos sistemas }\end{array}$ & 10 & 1 & 11 \\
\hline $\begin{array}{c}\text { Boa ou total } \\
\text { integração dos } \\
\text { sistemas }\end{array}$ & 11 & 11 & 22 \\
\hline Total & 21 & 12 & 33 \\
\hline
\end{tabular}

Para esta análise, achou-se $o$ valor de $p_{1}=0,04009$. Como nesta etapa 0 valor da menor frequência esperada também foi maior que zero, a modificação de Tocher foi realizada na tabela de frequências, conforme pode ser constatado na Tabela 6:

Tabela 6 - Frequências observadas para o tipo de ganho com inovação associado ao nível de integração dos sistemas de informação das empresas pernambucanas com a modificação de Tocher

\begin{tabular}{|c|c|c|c|}
\hline & $\begin{array}{c}\text { Pequeno ou médio } \\
\text { ganho com inovação }\end{array}$ & $\begin{array}{c}\text { Grande ou enorme } \\
\text { ganho com inovação }\end{array}$ & Total \\
\hline $\begin{array}{c}\text { Nenhuma, pouca ou } \\
\text { razoável integração } \\
\text { dos sistemas }\end{array}$ & 11 & 0 & 11 \\
\hline $\begin{array}{c}\text { Boa ou total } \\
\text { integração dos } \\
\text { sistemas }\end{array}$ & 10 & 12 & 22 \\
\hline Total & 21 & 12 & 33 \\
\hline
\end{tabular}

A partir da nova tabela, o valor de probabilidade $p_{2}$ calculado foi de 0,00334 , sendo, assim, $p_{t}$ igual a 0,04343, e portanto, maior que $o \alpha$ adotado. Desta forma, mais uma vez a hipótese nula foi rejeitada, indicando que há relação entre o nível de integração com os ganhos em inovação dos investimentos em TIC.

\subsection{Relação entre integração dos sistemas existentes com compartilhamento de dados e aplicações versus ganho com controle de atividades com base nos investimentos feitos no ano anterior em TIC}

Esta etapa consistiu na análise da relação entre as variáveis "nível de integração dos sistemas existentes com compartilhamento de dados e aplicações" e "nível de ganho com controle de atividades com base nos investimentos feitos no ano anterior em TIC". As hipóteses estabelecidas foram as seguintes: 
- $\mathrm{H}_{0}$ : o nível de integração de sistemas de informação não interfere no ganho com controle de atividades;

- $\mathrm{H}_{1}$ : a integração de sistemas de informação influencia o ganho com controle de atividades.

A Tabela 7 consta das frequências observadas para as amostras desta etapa, conforme é apresentado a seguir.

Tabela 7 - Frequências observadas para o tipo de ganho com controle de atividades associado ao nível de integração dos sistemas de informação das empresas pernambucanas

\begin{tabular}{|c|c|c|c|}
\hline & $\begin{array}{c}\text { Pequeno ou médio } \\
\text { ganho com controle } \\
\text { de atividade }\end{array}$ & $\begin{array}{c}\text { Grande ou enorme } \\
\text { ganho com controle } \\
\text { de atividades }\end{array}$ & Total \\
\hline $\begin{array}{c}\text { Nenhuma, pouca ou } \\
\text { razoável integração } \\
\text { dos sistemas }\end{array}$ & 7 & 4 & 11 \\
$\begin{array}{c}\text { Boa ou total } \\
\text { integração dos } \\
\text { sistemas }\end{array}$ & 7 & 15 & 22 \\
\hline Total & 14 & 19 & 33 \\
\hline
\end{tabular}

Aqui, com a aplicação da prova de Fisher, o valor calculado de probabilidade $p_{1}$ foi igual a 0,06873. Sendo este valor maior que o $\alpha$ de 0,05, a hipótese nula não pôde ser rejeitada, mesmo sem a necessidade de aplicação da modificação de Tocher e calculo de $\mathrm{p}_{2}$. Desta forma, não há indícios suficientes que nos leva a crer que a integração de Sls interfere nos ganhos com controle de atividades pelos dados obtidos.

\subsection{Relação entre integração dos sistemas existentes com compartilhamento de dados e aplicações versus ganho com redução de custos com base nos investimentos feitos no ano anterior em TIC}

A última etapa considerou as variáveis: "nível de integração dos sistemas existentes com compartilhamento de dados e aplicações" e "nível de ganho com controle de atividades com base nos investimentos feitos no ano anterior em TIC", tendo como hipóteses as apresentadas a seguir:

- $\mathrm{H}_{0}$ : o nível de integração de sistemas de informação não interfere no ganho com redução de custos; 
- $\mathrm{H}_{1}$ : a integração de sistemas de informação influencia o ganho com redução de custos.

A Tabela 8 apresenta as frequências para as amostras desta etapa.

Tabela 8 - Frequências observadas para o tipo de ganho com redução de custos associado ao nível de integração dos sistemas de informação das empresas pernambucanas

\begin{tabular}{|c|c|c|c|}
\hline & $\begin{array}{c}\text { Pequeno ou médio } \\
\text { ganho com redução } \\
\text { de custos }\end{array}$ & $\begin{array}{c}\text { Grande ou enorme } \\
\text { ganho com redução } \\
\text { de custos }\end{array}$ & Total \\
\hline $\begin{array}{c}\text { Nenhuma, pouca ou } \\
\text { razoável integração } \\
\text { dos sistemas }\end{array}$ & 8 & 3 & 11 \\
\hline $\begin{array}{c}\text { Boa ou total } \\
\text { integração dos } \\
\text { sistemas }\end{array}$ & 14 & 8 & 22 \\
\hline Total & 22 & 11 & 33 \\
\hline
\end{tabular}

Por meio da prova de Fisher, encontrou-se $p_{1}=0,27262$. Aqui, também, como $\mathrm{p}_{1}<\alpha$, a hipótese nula não é rejeitada, sem ser preciso aplicar a modificação de Tocher e calculo de $p_{2}$. Não há, portanto, evidências suficientes para crer que a integração de Sls interfere nos ganhos com redução de custos.

\section{DISCUSSÃO DOS RESULTADOS}

Através dos resultados identificados, pode-se inferir que o nível de integração dos sistemas de informação interfere em alguns ganhos obtidos com investimentos realizados em TIC, a saber: compreensão do negócio, aprendizados dos funcionários e inovação, cujos testes levaram à rejeição das hipóteses nulas. A razão disto consiste no fato da integração oferecer os requisitos que dão suporte para que tais ganhos sejam observados. Apesar disto, outras relações não puderam ser inferidas através dos testes realizados, como no caso dos ganhos com controle de atividades e redução de custos, nos quais a hipótese nula não foi rejeitada.

De maneira geral, as motivações que levam os gestores de TIC a realizarem investimentos são a redução de custos, aumento de qualidade, ganho em flexibilidade, entre outros (KHALLAF, 2012). Neste estudo, entretanto, foi possível identificar apenas que alguns desses ganhos (compreensão do negócio, aprendizado dos funcionários e inovação) apresentam relações com o nível de integração de sistemas de informação. 
A alta integração de sistemas de informação permite às organizações se tornarem capazes de coordenar de maneira mais eficiente suas operações e recursos, como também, aprimorarem seus relacionamentos com elementos externo, como os clientes, por exemplo, e, no caso da integração entre empresas, enxergar melhor a cadeia de suprimentos (LI et al., 2005). Desta forma, a integração amplia o campo de visão das empresas no que tange aos seus negócios, criando, assim, um ambiente propício para as estratégias organizacionais. Portanto, quando investimentos são realizados em ambientes sem tal suporte, os mesmos podem se tornar ineficazes no que diz respeito aos ganhos com compreensão do negócio uma vez que as empresas encontrarão barreiras para que conheçam como um todo as suas atividades, operações, clientes, mercados, entre outros. Ainda neste sentido, o baixo nível de integração pode fazer com que as informações fiquem partidas ou limitadas em cada área funcional ou organização, conforme aportando por Varga e Ćurko (2012), gerando, assim, isolamento, total ou parcial, das mesmas, que passam a não dispor de informações que poderiam ser úteis e estratégicas.

A integração de Sls também confere suporte aos ganhos com aprendizado dos funcionários no sentido de que permite o compartilhamento mais eficiente de dados, informações e conhecimento (VARGA; ĆURKO, 2012; SUN; TENG, 2012; OLESEN; MYERS, 1999; KLEIN; TELLEFSEN; HERSKOVITZ, 2007). Este compartilhamento é benéfico, pois, dentre tantas outras coisas, contribui para o ganho em eficiência, gerando aumento de sinergia e influenciando na redução de erros (CHEN; PAULRAJ, 2004; VICKERY et al., 2003). Assim, quando investimentos são feitos tendo foco na aprendizagem dos funcionários e são amparados por um ambiente com boa integração de sistemas de informação, estes podem se tronar bem-sucedidos com seus resultados alavancados.

Por fim, a inovação também é alcançada pelos recursos de TIC, sendo amparada pelos mesmos, os quais, nos últimos anos, têm levado muitas empresas a desenvolverem modelos de negócio inovadores. A isso, está associado, ainda, o fato das mudanças no ambiente ao qual a organização está inserida forçar a sua contínua inovação como estratégia de sobrevivência e, diante de tal situação, os SIs contribuem, uma vez que proporcionam melhor comunicação com o ambiente externo e a conexão e mobilização dos recursos internos da empresa (MERALI; PAPADOPOULOS; NADKARNI, 2012; TAYLOR, DILLON; VAN WINGEN, 2010). 
Sendo assim, como a integração dos Sls é capaz de potencializar as relações de conexão, comunicação e mobilização dentro da empresa que se refletem nos ganhos com compreensão do negócio e aprendizado dos funcionários, a integração acaba potencializando a inovação, também, seja no que diz respeito a projetos internos de novos processos, como no que tange a novos produtos ou serviços ofertados. Portanto, de maneira análoga aos ganhos citados anteriormente, a integração de Sls atua como agente facilitador criando o ambiente propício para que os investimentos realizados na área de TIC tragam como retorno ganhos em inovação.

Por sua vez, demais ganhos associados à integração de Sls também encontram amparo na literatura, como é o caso de redução de custos (CHANG; ITNER; PAZ, 2014; CHEN; CHIANG, 2011; MAIGA, 2015; SUN; IFEANYI, 2015) e controle de atividades (CHANG; ITNER; PAZ, 2014; TRUONG et al., 2011; WARING; SKOUMPOPOULOU, 2012). Contudo, no presente estudo, tais constatações não puderam ser confirmadas com os dados obtido de empresas pernambucanas, conforme apresentado em 5.4 e 5.5 .

\section{CONCLUSÃO}

Frente à mercados cada vez mais competitivos, a busca por melhores resultados no que se refere a desempenho organizacional consiste em um dos principais objetivos das empresas. Uma opção para o alcance deste consiste nos investimentos em TIC. Contudo, os desafios que tais apresentam fazem com que o estudo nesta área seja de grande valia a fim de compreender em que nível e sobre quais ganhos os investimentos em TIC são eficazes.

Este trabalho buscou, portanto, identificar a influência da integração dos sistemas de informação sobre alguns retornos obtidos com investimentos na área de TIC dentro de organizações localizadas no estado de Pernambuco. Por meio de testes de hipótese não-paramétricos realizados com a aplicação da prova de Fisher e modificação de Tocher, constatou-se que as organizações que apresentam nenhum, baixos ou médios níveis de integração de Sls obtiveram baixos retornos sobre investimentos no que tange a compreensão do negócio, aprendizados dos funcionários e inovação. Por sua vez, as empresas que dispunham de bons ou 
ótimos níveis de integração alcançaram melhores resultados. Tal constatação indica a importância da integração de Sls como característica estratégica para as organizações. Apesar de tais evidências, outras relações, contudo, não puderam ser inferidas pelos testes a fim de avaliar o impacto de tal opção de investimento em TIC no que diz respeito a controle de atividades e redução de custos.

Dentre os benefícios que a integração de Sls proporciona, pode-se destacar o compartilhamento mais eficiente de informações, gerando ampliação da percepção das empresas quanto a seus negócios, como também, aumento da sinergia entre áreas funcionais de uma organização ou entre organização membros da cadeia de suprimentos, isso porque a integração pode ser feita entre os sistemas de uma mesma empresa, entre sistemas de diferentes empresas, ou ambos os casos. Assim, a integração é capaz de aumentar a eficiência e eficácia das atividades e processos, na medida em que cria uma estrutura e ambiente adequados para que objetivos estratégicos das organizações sejam alcançados com maior êxito. Desta forma, sendo objetivos da integração de Sls, dentre outras coisas, ganhos com compreensão do negócio, aprendizado dos funcionários e inovação, estes acabam sendo influenciados, também, pelo nível de integração existente ao ponto de poderem ser limitados ou potencializados, uma vez que, apesar da integração ser considerada elemento estratégico para as organizações, ela sozinha não é suficiente.

Portanto, com o amparo da literatura associada, o presente trabalho identificou novas evidências acerca dos impactos da integração de Sls dentro das organizações, reforçando o seu papel como elemento estratégico, realizando, para tanto, análises entre os retornos oferecidos pelos investimentos em TIC em empresas com níveis distintos de integração de seus sistemas. Entretanto, apesar de sua contribuição, o mesmo apresenta limitações, principalmente pelo fato de contar com dados de empresas apenas do estado de Pernambuco. Outros estudos, contudo, deverão ser realizados a fim de compreender melhor as influências da integração de Sls sobre o desempenho organizacional, bem como, fatores de impacto sobre investimentos realizados na área de TIC. 


\title{
AGRADECIMENTOS
}

\author{
Este trabalho foi apoiado pelo Centro Nacional de Desenvolvimento Científico \\ e Tecnológico (CNPq).
}

\section{REFERÊNCIAS}

ARSHAH, R. A.; DESA, M. I.; HUSSIN, A. R. C. The need of Information Systems (IS) Integration Complexity Model for IS Integration Project. In: INTERNATIONAL SYMPOSIUM ON INFORMATION TECHNOLOGY, 4., 2008, Kuala Lumpur. Proceedings of the International Symposium on Information Technology, Kuala Lumpur: IEEE, 2008. p.1676-1684. http://dx.doi.org/10.1109/ITSIM.2008.4632005

AZADEH, A.; KERAMATI, A.; SONGHORI, M. J. An integrated Delphi/VAHP/DEA framework for evaluation of information technology/information system (IT/IS) investments. The International Journal of Advanced Manufacturing Technology, v. 45, n. 1, p. 1233-1251, dez. 2009. http://dx.doi.org/10.1007/s00170-009-2047-2

BALASUBRAMANIAN, P.; KULATILAKA, N.; STORCK, J. Managing information technology investments using a real-options approach. Journal of Strategic Information Systems, v. 9, n. 1, p. 39-62, mar. 2000. https://doi.org/10.1016/S0963-8687(00)00038-X

BEAL. A. Segurança da informação: princípios e as melhores práticas para a proteção dos ativos de informações nas organizações. 1. ed. São Paulo: Atlas, 2008. 176 p.

BHARADWAJ, A. S.; BHARADWAJ, S. G.; KONSYNSKI, B. Information technology effects on firm performance as measured by Tobin's Q. Management Science, v. 45, n. 7, p. 1008-1024, jul. 1999. http://dx.doi.org/10.1287/mnsc.45.7.1008

BROWN, M. M. Revisiting the IT Productivity Paradox. The American Review of Public Administration, v. 45, n. 5, p. 565-583, set. 2015. http://dx.doi.org/10.1177/0275074014523102

BRYNJOLFSSON, E. The productivity paradox of information technology. Communications of the ACM, v. 36, n. 12, p. 67-77, jan. 1993. http://dx.doi.org/10.1145/163298.163309

CHANG, H.; ITTNER, C. D.; PAZ, M. T. The multiple roles of the finance organization: Determinants, effectiveness, and the moderating influence of information system integration. Journal of Management Accounting Research, v. 26, n. 2, p. 1-32, abr. 2014. http://dx.doi.org/10.2308/jmar-50802

CHAROEN, D. Implementing integrated information systems project for police stations in Thailand. Journal of Cases on Information Technology, v. 17, n. 2, p. 14-34, abr. 2015. http://dx.doi.org/10.4018/JCIT.2015040102

CHEN, W. H.; CHIANG, A. H. Network agility as a trigger for enhancing firm performance: A case study of a high-tech firm implementing the mixed channel strategy. Industrial Marketing Management, v. 40, n. 4, p. 643-651, maio 2011. http://dx.doi.org/10.1016/j.indmarman.2011.01.001

CHEN, I. J.; PAULRAJ, A. Understanding supply chain management: critical research and theoretical framework. International Journal of Production Research, v. 42, n. 1, p. 131-163, 
Jan. 2004. http://dx.doi.org/10.1080/00207540310001602865

CHOU, Tzy-Yuan, CHOU, Seng-cho T. and TZENG, Gwo-Hshiung. Evaluating IT/IS investments: A fuzzy multi-criteria decision model approach. European Journal of Operational Research, v. 173, n. 3, p. 1026-1046, set. 2006. http://dx.doi.org/10.1016/j.ejor.2005.07.003

DARMAWAN RAYMOND, D. Engineering of automotive painting process using integrated information system to improve total painting and supply chain performance of paint in Indonesia. ARPN Journal of Engineering and Applied Sciences, v.10, n. 18, p. 8115-8120, out. 2015.

HAJLI, Mahmood; SIMS, Julian, M. Information technology (IT) productivity paradox in the 21st century. International Journal of Productivity and Performance Management, v. 64, n. 4, p. 457-478, abr. 2015. http://dx.doi.org/10.1108/lJPPM-12-2012-0129

HAMMER, Michael. The superefficient company. Harvard Business Review, Boston, v. 79, p. 82-91, set. 2001.

JOSHI, K.; PANT, S. Development of a framework to assess and guide IT investments: An analysis based on a discretionary-mandatory classification. International Journal of Information Management, v. 28, n. 3, p. 181-193, jun. 2008.

http://dx.doi.org/10.1016/j.ijinfomgt.2007.09.002

KAPLAN, R. S.; NORTON, D. P. Transforming the balance scorecard from performance measurement to strategic performance: Part 1. Accounting Horizons, v. 15, n. 1, p. 87-104, Mar. 2001. http://dx.doi.org/10.2308/acch.2001.15.1.87

KHALLAF, Ashraf. Information technology investments and nonfinancial measures: A research framework. Accounting Forum, v. 36, n. 2, p. 109-121, jun. 2012.

http://dx.doi.org/10.1016/j.accfor.2011.07.001

KLEIN, E. E.; TELLEFSEN, T.; HERSKOVITZ, P. J. The use of group support systems in focus groups: Information technology meets qualitative research. Computers in Human Behavior, v. 23, n. 5, p. 2113-2132, set. 2007. http://dx.doi.org/10.1016/j.chb.2006.02.007

LI, S.; RAO, S. S.; RAGU-NATHAN, B.; RAGU-NATHAN., T. S. Development and validation of a measurement instrument for studying supply chain management practices. Journal of

Operations Management, v. 23, n. 6, p. 618-641, set. 2005.

http://dx.doi.org/10.1016/j.jom.2005.01.002

LI, H.; SU, S. Y. W. Business Object Modeling, Validation, and Mediation for Integrating Heterogeneous Application Systems. Journal of Systems Integration, v. 10, n. 4, p. 307-328, set. 2001. http://dx.doi.org/10.1023/A:1011246500072

LITAN, D.; VELICANU, M.; COPCEA (Teohari), L.; TEOHARI, M.; MOCANU (Virgolici), A. M.; SURUGIU, I.; RADUTA, O. Business' New Requirement: Information Systems Integration Methods and Technologies. International Journal of Computers and Communications, v. 5, n. 3, p. 133-145, 2011.

MAIGA, A. S. Information Systems Integration and Firm Profitability: Mediating Effect of Cost Management Strategy. Advances in Management Accounting, v. 25, p. 149-179, 2015. http://dx.doi.org/10.1108/S1474-787120150000025004

MAIGA, A. S.; NILSSON, A.; AX, C. Relationships between internal and external information systems integration, cost and quality performance, and firm profitability. International Journal of Production Economics, v. 169, p. 422-434, nov. 2015.

http://dx.doi.org/10.1016/j.ijpe.2015.08.030 
MERALI, Y.; PAPADOPOULOS, T.; NADKARNI, T. Information systems strategy: Past, present, future? Journal of Strategic Information Systems, v. 21, n. 1, p. 125-153, Jun. 2012. https://doi.org/10.1016/j.jsis.2012.04.002

MIRANI, R.; LEDERER, A. L. An instrument for assessing the organizational benefits of IS projects. Decision Sciences Institute, v. 29, n. 4, p. 803-838, set. 1998.

http://dx.doi.org/10.1111/j.1540-5915.1998.tb00878.x

MÖLLER, E. B.; FRANK, A. G.; CORTIMIGLIA, M. N. Critérios e fatores críticos de sucesso para a implantaçÃo de sistemas de informação: um estudo de caso sob a perspectiva de uma empresa implantadora de sistemas. Revista produção Online, v. 14, n. 4, p. 1378-1403, out./dez. 2014. http://dx.doi.org/10.14488/1676-1901.v14i4.1681

MÖLLER, E. B.; SCHATTSCHNEIDE, G. O.; FRANK, A. G.; RIBEIRO, J. L. D. Qualidade da informação no pcp: análise dos fatores de influência e proposta de um método de diagnóstico. Revista Produção Online, v. 13, n. 1, p. 37-60, jan./mar. 2013. http://dx.doi.org/10.14488/16761901.v13i1.1026

OLESEN, K.; MYERS, Michael D. Trying to improve communication and collaboration with information technology: An action research project which failed. Information Technology and People, v. 12, n. 4, p. 317-332, dez. 1999. http://dx.doi.org/10.1108/09593849910301621

PERERA, M. U. S. Decision Making Delays with Regard to IT Investments. Procedia - Social and Behavioral Sciences, v. 40, n. 1, p. 258-263, 2012.

http://dx.doi.org/10.1016/j.sbspro.2012.03.188

RAJAGURU, R.; MATANDA, M. J. Effects of inter-organizational compatibility on supply chain capabilities: Exploring the mediating role of inter-organizational information systems (IOIS) integration. Industrial Marketing Management, v. 42, n. 1, p. 620-632, maio 2013. http://dx.doi.org/10.1016/j.indmarman.2012.09.002

ROBERTS, N.; GROVER, V. Leveraging Information Technology Infrastructure to Facilitate a Firm's Customer Agility and Competitive Activity: An Empirical Investigation. Journal of Management Information Systems, v. 28, n. 4, p. 231-270, abr. 2012. http://dx.doi.org/10.2753/MIS0742-1222280409

SHANAHAN, C. W.; SORENSEN-ALAWAD, A.; CARNEY, B. L.; PERSAND, I.; CRUZ, A.; BOTTICELLI, M.; PRESSMAN, K.; ADAMS, W. G.; BROLIN, M.; ALFORD, D. P. The implementation of an integrated information system for substance use screening in general medical settings. Applied Clinical Informatics, v. 5, n. 4, p. 878-894, out. 2014. http://dx.doi.org/10.4338/ACl-2014-03-RA-0025

SILVA, A. L. C. L.; DAHER, S. F. D.; SILVA, L. C. Uma análise exploratória sobre os ganhos obtidos com os investimentos em TIC por empresas pernambucanas. In: ENCONTRO NACIONAL DE ENGENHARIA DE PRODUÇÃO, 35., 2015, Fortaleza. Anais eletrônicos da Associação Brasileira de Engenharia de Produção. Fortaleza: ABEPRO, 2015. Disponível em: <http://www.abepro.org.br/biblioteca/TN_WIC_213_263_27762.pdf >. Acesso em: 10 jan. 2017.

SILVA, A. L. C. L.; DAHER, S. F. D.; SILVA, L. C. Investigação sobre a percepção de gestores quanto aos benefícios dos investimentos em TI. In: ENCONTRO NACIONAL DE ENGENHARIA DE PRODUÇÃO, 36., 2016, João Pessoa. Anais eletrônicos da Associação Brasileira de Engenharia de Produção. Paraíba: ABEPRO, 2016. Disponível em: <http://www.abepro.org.br/biblioteca/TN_WIC_233_360_29971.pdf>. Acesso em: 07 jan. 2017. 
SOLOW, R. M. We'd better watch out. The New York Times Book Review, New York, v. 12, n.1, p. 36, 12 jul. 1987.

SUN, Y.; IFEANYI, O. A qualitative study of e-business adoption in the real estate sector in China. Journal of Internet Banking and Commerce, v. 20, n. 1, p. 1-10, abr. 2015.

SUN, J.; TENG, J. T.C. Information Systems Use: Construct conceptualization and scale development. Computers in Human Behavior, v. 28, n. 5, p. 1564-1574, set. 2012. http://dx.doi.org/10.1016/j.chb.2012.03.016

TAYLOR, H.; DILLON, S.;VAN WINGEN, M. Focus and diversity in information systems research: meeting the dual demands of a healthy applied discipline. MIS Quarterly: Management Information Systems, v. 34, n. 4, p. 647-667, dez. 2010.

TOMICIC-PUPEK, K.; DOBROVIC, Z.; FURJAN, M. T. Strategies for Information Systems Integration. In: INTERNATIONAL CONFERENCE ON INFORMATION TECHNOLOGY INTERFACES, 34., 2012, Cavtat. Proceedings of the International Conference on Information Technology Interfaces. Cavtat: SRCE UNIV., 2012. p. 311-316. http://dx.doi.org/10.2498/iti.2012.0459

TRUONG, C. V. C.; GROENEVELD, L. F.; MORGENSTERN, B.; GROENEVELD, E. MolabIS An integrated information system for storing and managing molecular genetics data. BMC

Bioinformatics, v. 12, n. 425, p. 1-15, out. 2011. http://dx.doi.org/10.1186/1471-2105-12-425

VARGA, M.; ĆURKO, K. Some Aspects of Information Systems Integration. In: INTERNATIONAL CONVENTION ON INFORMATION AND COMMUNICATION TECHNOLOGY, ELECTRONICS AND MICROELECTRONICS, 35., 2012, Opatija. Proceedings of the International Convention on Information and Communication Technology, Electronics and Microelectronics, Opatija: MIPRO, 2012. p. 1583-1588.

VICKERY, S. K.; JAYARAM, J.; DROGE, C.; CALATONE, R. The effects of an integrative supply chain strategy on customer service and financial performance: An analysis of direct versus indirect relationships. Journal of Operations Management, v, 21, n. 5, p. 523-539, dez. 2003. http://dx.doi.org/10.1016/i.jom.2003.02.002

WARING, T.; SKOUMPOPOULOU, D. An enterprise resource planning system innovation and its influence on organisational culture: A case study in higher education. Prometheus, v. 30, n. 4, p. 427-447, dez. 2012. http://dx.doi.org/10.1080/08109028.2012.754572

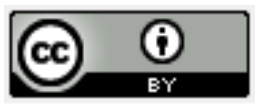

Artigo recebido em 23/02/2017 e aceito para publicação em 11/08/2017 DOI: http://dx.doi.org/10.14488/1676-1901.v17i3.2759 